\title{
Acute Small Bowel Obstruction due to Intra-Abdominal Lithopedion
}

\section{Salako Alexandre Allodé ${ }^{1}$, Emile Mensah ${ }^{2}$ and Francis Moïse Dossou ${ }^{3 *}$}

${ }^{1}$ Clinique universitaire de chirurgie générale, Centre hospitalier départemental et universitaire de Parakou, Bénin ${ }^{2}$ Clinique universitaire de chirurgie générale, Centre hospitalier départemental et universitaire de Parakou, Bénin

${ }^{3}$ Clinique universitaire de chirurgie viscérale B, Centre national hospitalier et universitaire Hubert Koutoukou Maga de Cotonou, Bénin

\begin{abstract}
A case report of a 32-year old woman who had 2 years ago a miscarriage pregnancy at 5 months of gestation and was admitted urgently for abdominal pain lasting for 72 hours. Pain was accompanied by nausea, vomiting, absence of stools and gas for 48 hours. The abdomen was distended. Acute intestinal obstruction was diagnosed. Straight radiograph confirmed the obstruction and located it on the small bowel. At laparotomy, there was an agglutination of small bowel loops around I lithopedion, cause of the acute bowel obstruction. The loops were dilated and there was a local ischemia due to a mesenteric torsion around the lithopedion. The lithopedion was released and an ileal resection with immediate anastomosis was performed. The postoperative course was uneventful and the patient was discharged from hospital on the $10^{\text {th }}$ postoperative day.
\end{abstract}

Keywords: Lithopedion; Acute bowel obstruction; Intestinal resection; Abdominal pregnancy; Sub-Saharan Africa

\section{Introduction}

The lithopedion is a dead fetus, infiltrated by calcium salt after a long stay in the womb or in the abdomen [1]. We report a case of abdominal lithopedion discovered during an acute bowel obstruction.

\section{Observation}

A 32 -year old woman gestity 2, parity 1 , with a history 2 years ago of a miscarriage pregnancy at 5 months of gestation was admitted urgently for abdominal pain lasting for 72 hours. The miscarriage was not followed by any operation to empty the uterus as the patient lived in a village located $320 \mathrm{Km}$ from our hospital and away from any qualified health center. There was a gradual regression of the volume of the abdomen and a resumption of normal menses. The patient had her last menstruation 11 days before her admission. Pain was accompanied by nausea, vomiting, absence of stools and gas for 48 hours. An abdominal distension completed the obstructive syndrome. Acute intestinal obstruction was diagnosed. The gynecological examination was normal with a closed cervix and painless and mid-size uterus. Straight radiograph confirmed the obstruction and located it on the small bowel by showing characteristic images (Figure 1). At laparotomy, the pelvis was occupied by an agglutination of dilated and ischemic small bowel loops around a mummified fetus (lithopedion). The lithopedion was in the pelvis and was not identified on the radiography because of the superposition of the pelvic bones. Membranes, also calcified, were attached to bowel and mesentery that turned around the lithopedion, provoking a mesenteric torsion. The lithopédion was released (Figure

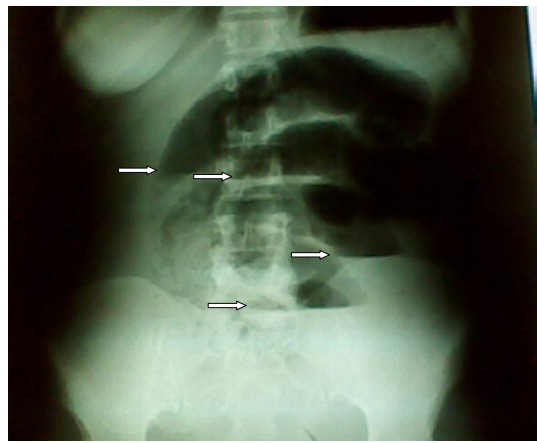

Figure 1: Straight abdominal radiograph showing characteristic images of small bowel obstruction (white arrows).
2) and an ileal resection about $1 \mathrm{~m}$ was performed followed by immediate ileo-ileal end to end anastomosis. The examination of the uterine wall and fallopian tubes was normal. The postoperative course was uneventful. The patient was discharge from hospital on the $10^{\text {th }}$ postoperative day. The morphology of lithopedion was clearly observed after spending 48 hours in a solution of formaldehyde (Figure 3).

\section{Comment}

Bowel obstruction is an exceptional consequence of lithopedion. Glass and Abramson in 1953 [2] reported the occurrence of volvulus of the cecum due to a lithopedion. The twist was caused by the

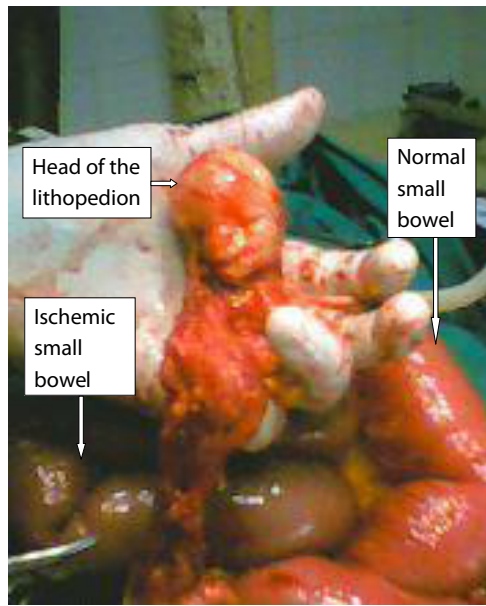

Figure 2: Dissection of the lithopedion.

${ }^{*}$ Corresponding author: Dr. Francis Moïse DOSSOU, Clinique universitaire de chirurgie viscérale $B$, Centre national hospitalier et universitaire Hubert Koutoukou Maga de Cotonou, 06 BP 939 Cotonou Benin, Tel : 00 (229) 9506 22 85; E-mail: dosfm@yahoo.fr

Received January 23, 2012; Accepted February 07, 2012; Published February 09,2012

Citation: Allodé SA, Mensah E, Dossou FM (2012) Acute Small Bowel Obstruction due to Intra-Abdominal Lithopedion. Surgery 2:107. doi:10.4172/2161 1076.1000107

Copyright: (C) 2012 Allodé SA, et al. This is an open-access article distributed under the terms of the Creative Commons Attribution License, which permits unrestricted use, distribution, and reproduction in any medium, provided the original author and source are credited. 


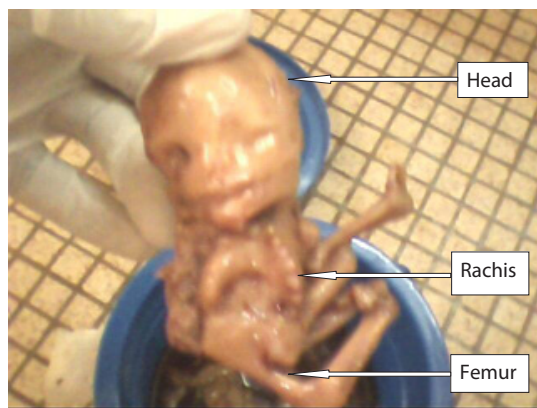

Figure 3: Lithopedion after spending 48 hours in a solution of formaldehyde.

adhesion of the ascending colon to a retrocolic lithopedion. Zaheer in 1971 relates the circumstances of occurrence of small bowel obstruction by intra-abdominal lithopedion [3]. Our case looks like Zaheer's, as the lithopedion adhered to the small bowel and caused its obstruction. It also was the cause of a mesenteric ischemia by the calcification of the placental membrane. Indeed, it was strictly speaking a lithocelyphopedion because the fetus and the placental membrane were calcified. The other two categories of lithopedion are lithocelyphos in which only the membranes are calcified but not the fetus and true lithopedion or lithoteknon, where only the child's body is covered and mummified by calcified films [4,5]. The lithopedions may have a long history evolving for 4 to 60 years in women from lower socioeconomic backgrounds with poor access to prenatal care $[4,6]$. They occur sometimes symptoms made of chronic pelvic pain, abdominal heaviness, compression syndromes affecting especially the urinary tract and rectum. They are usually asymptomatic, discovered incidentally by visualizing the lithopedion on a straight abdominal radiograph $[4,5]$. In our case, the evolution was cut short by the occurrence of acute small bowel obstruction that led to discover the lithopedion at emergent laparotomy. Indeed, the lithopedion was not clearly demonstrated on radiographs because of its small size and the superposition of the pelvic bones.
The surgical management of lithopedion is usually simple with very positive course even in the elderly [5,7]. We performed a resection of the ischemic small bowel loops and anastomosis. The postoperative course was favorable in our case, but we know that the sutures have their own digestive disease characterized by the occurrence sometimes of intestinal fistula after surgery [8]. To our knowledge, no intraoperative death due to lithopedion has been reported and the only death reported in the literature is not related to lithopedion, but the patient died of pulmonary embolus after a formal above-knee amputation performed for a leg dry gangrene [5].

\section{Conclusion}

Abdominal pregnancy is rare and exceptionally ends by a lithopedion which may cause so rarely an acute intestinal obstruction. The surgical management is easy but the lithopedion remains an entity which should completely disappear with the antenatal consultation and the early diagnosis and appropriate treatment of abdominal pregnancy.

\section{Références}

1. Garnier M, Delamare V, Delamare J, Delamare T, Delamare F (2009) Dictionnaire illustré des termes de médecine. (30édition), Paris: 514

2. Zaheer SA (1971) Acute intestinal obstruction caused by lithopedion. Br J Surg 58: 401-402.

3. Glass BA, Abramson PD (1953) Volvulus of cecum due to lithopedion. Am J Surg 86: 348-352.

4. Júnior RP, Knobel R, Parpinelli MA, Pereira BG, Amaral E, et al. (2000) Calcified abdominal pregnancy with eighteen years of evolution: case report Sao Paulo Med J/Rev Paul Med 118: 192-194.

5. Ede J, Sobnach S, Castillo F, Bhyat H, Corbett JH (2011) The lithopedion - an unusual cause of an abdominal mass. S Afr J Surg 49: 140-141.

6. Adéhossi E, Gbaguidi F, Garba M, Kaba-Cissé MF, Parola P (2006) Lithopédion. Med Trop 66: 30 .

7. Kim MS, Park S, Lee TS (2002) Old abdominal pregnancy presenting as an ovarian neoplasm. J Korean Med Sci 17: 274-275.

8. Harouna Y, Yaya H, Abdou I, Bariza L (2000) Pronostic de la hernie inguinale étranglée de l'adulte : influence de la nécrose intestinale. A propos de 34 cas. Bull Soc Pathol Exot 93: 317-320. 